\title{
Strates
}

STRATES Matériaux pour la recherche en sciences sociales

$10 \mid 2001$

Villageois et citadins de Grèce

\section{Relations familiales et sociabilité en milieu urbain: le cas du Pirée}

Laura Maratou-Alipranti et Andromachi Hadjiyanni

\section{(2) OpenEdition \\ 1 Journals}

Édition électronique

URL : http://journals.openedition.org/strates/370

DOI : $10.4000 /$ strates. 370

ISSN : $1777-5442$

Éditeur

Laboratoire Ladyss

Édition imprimée

Date de publication : 1 mai 2001

ISSN : 0768-8067

Référence électronique

Laura Maratou-Alipranti et Andromachi Hadjiyanni, «Relations familiales et sociabilité en milieu urbain: le cas du Pirée », Strates [En ligne], 10 | 2001, mis en ligne le 11 janvier 2005, consulté le 10 décembre 2020. URL : http://journals.openedition.org/strates/370 ; DOI : https://doi.org/10.4000/ strates.370

Ce document a été généré automatiquement le 10 décembre 2020.

Tous droits réservés 


\title{
Relations familiales et sociabilité en milieu urbain : le cas du Pirée
}

\author{
Laura Maratou-Alipranti et Andromachi Hadjiyanni
}

1 En Grèce, la population des villes a augmenté de façon brusque, en quelques dizaines d'années. Juste après-guerre, la plus grande partie de la population vivait encore dans des régions rurales et exerçait une profession agricole. Ce bouleversement rapide a pour conséquence la persistance en milieu urbain de modes de vie sociale comparables à ceux du monde rural. Sont caractéristiques de cette sociabilité : les rapports étroits d'inter-connaissance et d'entraide au sein de la communauté, les liens familiaux étroits, mais aussi l'existence et le fonctionnement de réseaux sociaux puissants, dans plusieurs domaines de la vie sociale, comme les réseaux de clientèle (Mouzelis, 1978, Tsoukalas, 1985, Damianakos, 1987).

2 Nos connaissances concernant les relations sociales en ville ainsi que l'ampleur de ces évolutions sont limitées. Les recherches en sociologie urbaine traitant des relations familiales et sociales ou, plus généralement, de sociabilité, furent, jusqu'aux années quatre-vingt, peu nombreuses. Cet article présente une partie des matériaux ${ }^{1}$ réunis lors des recherches de sociologie urbaine réalisées dans le cadre d'une collaboration franco-hellénique entre 1986 et 1992 (Cnrs, Ekke grec et université de Paris XNanterre). L'enquête empirique s'est déroulée dans quatre quartiers de la ville du Pirée, trois quartiers ouvriers et un quartier petit-bourgeois ${ }^{2}$, auprès d'un échantillon représentatif de huit cents individus (dont la moitié de femmes), d'âge compris entre 20 et 59 ans. Menée par questionnaire, cette investigation visait à recueillir des données portant essentiellement sur la famille, la mobilité socio-professionnelle et l'emploi. L'analyse présentée ici est plus particulièrement centrée sur deux points : d'une part, les formes de relations nouées entre générations à l'intérieur de la famille et d'autre part, les types de sociabilité qui se développent au niveau de la localité.

Quelques hypothèses

3 Les quartiers ou communes de la région du Pirée sur lesquels porte notre recherche font partie du Grand Athènes, c'est-à-dire du plus grand centre urbain du pays. On a donc présumé que : 
4 1. les relations sociales des habitants au niveau local seraient limitées et d'importance relative ;

2. les migrants n'auraient plus de relation avec leur lieu d'origine ;

6 3. le fort pourcentage de "migrants de l'intérieur " aurait des conséquences négatives sur la sociabilité au niveau local. Ces individus seraient en rupture avec leurs réseaux familiaux et sociaux ;

7 4. sachant que le pourcentage de gens qui cohabitent avec leurs parents est devenu peu important (la famille nucléaire est actuellement le modèle dominant dans l'espace urbain) les relations entre les membres d'une même famille seraient moins étroites et les pratiques d'aide limitées.

I. Installation en milieu urbain

8 L'exode rural des années soixante et soixante-dix et l'installation des migrants en milieu urbain, essentiellement à Athènes, furent un phénomène social majeur aux multiples conséquences sociales (Kotzamanis, 1997).

9 Les personnes originaires de la région de la capitale représentent $57 \%$ des personnes interviewées, et les migrants les $43 \%$ restants (dans deux quartiers, les migrants de première génération représentent la moitié de la population).

Quelles sont les raisons justifiant la présence de migrants et non-migrants dans un quartier donné ? Un fort pourcentage d'entre eux évoquent la présence de leur famille : leur famille d'origine ou celle de leur conjoint, ou celle de membres plus éloignés ; tous sont logés gratuitement par elle... (tableau 1). Ce phénomène est associé aux relations qui existent entre parents proches et même entre parents plus éloignés. Les contacts sont fréquents; la sociabilité des migrants, on le verra, est en grande partie tournée vers la famille.

Tableau 1 : Raisons évoquées par l'enquêté pour expliquer sa présence dans un quartier donné selon son lieu d'origine (\% en colonne)

\begin{tabular}{|c|c|c|c|c|c|c|}
\hline & \multicolumn{6}{|c|}{ Lieu d'origine } \\
\hline \multirow{2}{*}{$\begin{array}{l}\text { Raisons } \\
\text { d'installation }\end{array}$} & \multicolumn{2}{|c|}{ Région de la capitale } & \multicolumn{3}{|c|}{ Autre lieu (province) } & Total \\
\hline & $\mathrm{N}$ & $\%$ & $\mathrm{~N}$ & $\%$ & & $\mathrm{~N} \mid \%$ \\
\hline Les parents y vivaient & 250 & 57,3 & 16 & 4,9 & 266 & 34,7 \\
\hline Il y avait de la famille & 10 & 2,3 & 59 & 17,9 & 69 & 9,0 \\
\hline Loyers bas & 4 & 0,9 & 5 & 1,5 & 9 & 1,2 \\
\hline Beau Quartier & 12 & 2,3 & 7 & 2,1 & 19 & 2,5 \\
\hline Hasard & 22 & 5,0 & 44 & 13,3 & 66 & 8,6 \\
\hline Le conjoint y vivait & 54 & 12,5 & 90 & 27,3 & 144 & 18,9 \\
\hline
\end{tabular}




\begin{tabular}{|l|l|l|l|l|l|l|}
\hline Il avait acheté un terrain & 9 & 2,1 & 12 & 3,6 & 21 & 2,7 \\
\hline \hline Près du travail & 25 & 5,7 & 61 & 18,5 & 86 & 11,2 \\
\hline \hline $\begin{array}{l}\text { La famille a prêté } \\
\text { un logement }\end{array}$ & 50 & 1,5 & 36 & 10,9 & 86 & 11,2 \\
\hline \hline Total & 436 & 100,0 & 330 & 100,0 & 766 & 100,0 \\
\hline
\end{tabular}

11 La majorité des migrants intérieurs est installée dans la région de la capitale depuis vingt, trente et même quarante ans. Pourtant, les liens qu'ils conservent avec leur lieu d'origine restent forts. Seules $37 \%$ des personnes interviewées considèrent la région d'Athènes comme leur lieu d'origine. Or, le pourcentage de ceux qui ont grandi dans la capitale s'élève à $57 \%$ : non seulement ceux qui ont grandi en province, mais également une part importante de ceux qui ont grandi dans la région d'Athènes se sentent originaires d'un autre lieu que la capitale. Cela prouve les rapports étroits qui les unissent avec leur lieu d'origine et constitue un indicateur de la résistance qu'ils opposent à l'intégration dans l'espace social urbain.

12 La conservation de propriétés d'une part et le maintien des droits de vote au lieu d'origine d'autre part montrent également la force des liens avec le lieu d'origine. $17,5 \%$ des personnes interviewées quittent leur lieu de résidence pour aller voter ailleurs lors des élections (ce pourcentage est plus élevé dans les quartiers où la présence des migrants est plus forte, comme à Kaminia: 22,5\%). Les relations de clientèle ou l'identification des Grecs à leur lieu d'origine contribuent à la pérennité de ce phénomène.

13 En examinant plus en détail la relation des migrants intérieurs avec leur lieu d'origine, on s'aperçoit qu'un très petit nombre d'entre eux (4\%) à la fois votent, se font recenser et se sentent originaires d'un autre lieu que la région d'Athènes, et sont ce qu'on pourrait appeler des provinciaux « purs ».

14 Les Piréotes ou Athéniens "purs », c'est-à-dire ceux qui n'ont aucun rapport avec un autre lieu que la capitale (voter, se faire recenser ou se sentir originaire), représentent quant à eux $35 \%$ des interviewés. Les habitants qui conservent une relation sociale (soit voter, soit se faire recenser) ou sentimentale (se sentir originaire) avec la province dont ils sont originaires, représentent presque les deux tiers de l'ensemble des personnes interviewées.

15 Notre hypothèse selon laquelle les migrants n'ont pas conservé de lien avec leur pays d'origine n'est donc pas confirmée. Au contraire, les migrants entretiennent des liens forts avec leur lieu d'origine, que ce soit les migrants intérieurs de la première génération ou de la seconde, c'est-à-dire des personnes qui sont nées et ont grandi dans la région de la capitale. On voit donc que l'identité de la personne n'est pas seulement associée au lieu où elle est née ou a grandi. Elle dépend aussi des représentations et des valeurs qui lui sont transmises.

II. Famille et sociabilité

16 Dans toutes les sociétés, la vie sociale des individus est étroitement liée à la famille. Les relations familiales composent une part plus ou moins grande de leurs relations 
sociales, comme le démontrent plusieurs recherches au niveau international (Forsé, 1991, 1993).

D'après nos résultats, les habitants des quartiers étudiés ont beaucoup d'activités communes avec leur parentèle. Qu'en est-il plus précisément de la fréquence des contacts entre les interviewés et leurs parents/beaux parents ainsi qu'avec les membres de leur fratrie?

La distance géographique du couple avec les deux «familles d'origine" (parents et beaux-parents) conditionne le rythme des contacts. Le tiers environ des personnes interrogées vit dans le même appartement ou le même immeuble qu'une des familles d'origine. Un autre tiers vit à proximité : dans le même quartier ou un autre quartier du Pirée. Dans $15 \%$ des cas, l'une ou les deux familles d'origine vivent en dehors d'Athènes.

En se mariant, une grande partie des couples du Pirée ne s'éloigne pas de leur famille. Ce fait est aussi lié au modèle et au processus d'urbanisation développé en Grèce après guerre qui ont sans doute eu une influence sur les choix résidentiels des ménages (Kayser, 1978; Kayser et al. 1971 ; Hadjiyannis, Maratou-Alipranti, 1995; Maloutas, 1995). Cette proximité avec les familles d'origine laisse supposer l'importance des contacts et des pratiques d'aide entre les deux générations.

a) La fréquence des rencontres

Les données du tableau 2 nous indiquent que dans les cas de co-résidence ou de proximité géographique (les deux ménages habitant le même quartier), $97 \%$ des ménages se rencontrent presque quotidiennement. Cependant, les contacts deviennent moins fréquents lorsque les parents s'éloignent, c'est-à-dire habitent dans une autre partie du Pirée ou du Grand Athènes. Même dans ce cas, plus de la moitié (53,2\%) se rencontrent plus d'une fois par semaine et $40 \%$ se voient au moins une fois par mois.

$21 \mathrm{Au}$ contraire, la fréquence des contacts diminue considérablement si les parents résident en dehors d'Athènes : $83,2 \%$ des couples les voient seulement une ou deux fois par an, pendant les vacances et les grandes fêtes. Cependant, $17 \%$ des époux dans cette situation rencontrent au moins une fois par mois leurs parents. Généralement, il s'agit de parents résidant dans les départements les plus proches de la capitale.

Tableau 2: Fréquence des rencontres des époux avec les parents et les beaux-parents en fonction du lieu de résidence (\% en ligne)

\begin{tabular}{|c|c|c|c|c|c|c|c|c|c|}
\hline \multirow{2}{*}{$\begin{array}{l}\text { Lieu de résidence } \\
\text { des parents }\end{array}$} & \multicolumn{3}{|c|}{$\begin{array}{l}\text { Plus d'une fois } \\
\text { par semaine }\end{array}$} & $\begin{array}{l}\text { 1-4 fois } \\
\text { par mois }\end{array}$ & \multicolumn{3}{|c|}{$\begin{array}{l}\text { 1-2 fois } \\
\text { par an/rarement }\end{array}$} & \multicolumn{2}{|c|}{ Total } \\
\hline & $\mathrm{N}$ & $\%$ & & $\%$ & $\mathrm{~N}$ & & & $\mathrm{~N}$ & $\%$ \\
\hline $\begin{array}{l}\text { Au moins un dans le même logement ou dans le } \\
\text { même immeuble ou dans le même quartier }\end{array}$ & 385 & 97,2 & 8 & 2,0 & 3 & 0,8 & 396 & & 9,2 \\
\hline $\begin{array}{l}\text { Au moins un dans la région du Pirée / } \\
\text { Grand Athènes }\end{array}$ & 82 & 53,2 & 61 & 39,6 & 11 & 7,2 & 154 & 23 & 3,0 \\
\hline
\end{tabular}




\begin{tabular}{|l|l|l|l|l|l|l|l|l|l|}
\hline Aucun dans le Grand Athènes & - & - & 20 & 16,8 & 99 & 83,2 & 119 & 17,8 \\
\hline \hline Total & 467 & 69,8 & 89 & 13,3 & 113 & 16,9 & $669^{*}$ & 100,0 \\
\hline
\end{tabular}

* Dans 97 cas, les parents/beaux-parents sont décédés

Tableau 3 : Fréquence des rencontres des enquêtés avec les parents et les beaux-parents selon leur origine géographique (\% en ligne)

\begin{tabular}{|c|c|c|c|c|c|c|c|c|}
\hline \multirow[t]{2}{*}{$\begin{array}{l}\text { Lieu de résidence } \\
\text { des parents }\end{array}$} & \multicolumn{2}{|c|}{$\begin{array}{l}\text { Plus d'une fois } \\
\text { par semaine }\end{array}$} & \multicolumn{2}{|c|}{$\begin{array}{l}\text { 1-4 fois } \\
\text { par mois }\end{array}$} & \multicolumn{2}{|c|}{$\begin{array}{l}1-2 \text { fois } \\
\text { par an/rarement }\end{array}$} & \multicolumn{2}{|l|}{ Total } \\
\hline & $\mathrm{N}$ & $\%$ & $\mathrm{~N}$ & $\%$ & $\mathrm{~N}$ & $\%$ & $\mathrm{~N}$ & $\%$ \\
\hline Les deux époux migrants & 60 & 37,5 & 26 & 16,3 & 74 & 46,2 & $\begin{array}{l}160 \\
{[23,9]}\end{array}$ & 100,0 \\
\hline Un des époux migrants & 184 & 74,8 & 33 & 13,4 & 29 & 11,8 & $\begin{array}{l}246 \\
{[36,8]}\end{array}$ & 100,0 \\
\hline Les deux d'Athènes & 223 & 84,8 & 30 & 11,4 & 10 & 3,8 & $\begin{array}{l}263 \\
{[39,3]}\end{array}$ & 100,0 \\
\hline Total & 467 & 69,8 & 89 & 13,3 & 113 & 16,9 & $\begin{array}{l}669^{*} \\
{[100,0]}\end{array}$ & 100,0 \\
\hline
\end{tabular}

* Dans 97 cas, les parents/beaux-parents sont décédés

L'origine géographique des époux conditionne la fréquence des rencontres entre les générations. Lorsque les deux époux sont migrants, les rencontres avec leurs parents sont évidemment assez rares : les rencontres ont lieu seulement une ou deux fois par an. Lorsque l'un ou les deux époux sont originaires d'Athènes, ils habitent fréquemment près de leur famille et entretiennent des relations fréquentes avec leurs parents ( $80 \%$ se voient plus d'une fois par semaine contre 37,5 pour les migrants) (tableau 3).

Quelle est la fréquence des rencontres des couples avec leurs collatéraux ? Elle dépend, comme pour les contacts avec les parents, de leur lieu de résidence.

La majorité des frères et sœurs des interviewés habite le Grand Athènes (70\%). Ils se voient souvent : $83 \%$ des couples rencontrent un frère/sœur au moins une fois par semaine, $14 \%$ au moins une fois par mois. Par contre, lorsque les membres de la fratrie sont un peu éloignés (dans un autre quartier de la capitale), les contacts sont moins aisés. Même dans ce cas, les deux tiers rencontrent leur frère ou leur sœur presque une fois par semaine. La distance agit de manière négative et les rencontres sont rares (une ou deux fois par an) lorsque le lieu de résidence des membres de la fratrie est en dehors d'Athènes (tableau 4). 
Tableau 4 : Fréquence des rencontres des enquêtés avec au moins un membre de la fratrie en fonction du lieu de résidence (\% en ligne)

\begin{tabular}{|c|c|c|c|c|c|c|c|c|}
\hline \multirow[t]{2}{*}{$\begin{array}{l}\text { Lieu de résidence } \\
\text { de la fratrie }\end{array}$} & \multicolumn{2}{|c|}{$\begin{array}{l}\text { Plus d'une } \\
\text { fois } \\
\text { par semaine }\end{array}$} & \multicolumn{2}{|c|}{$\begin{array}{l}\text { 1-4 fois } \\
\text { par mois }\end{array}$} & \multicolumn{2}{|c|}{$\begin{array}{l}\text { 1-2 fois } \\
\text { par an/ } \\
\text { rarement }\end{array}$} & \multicolumn{2}{|l|}{ Total } \\
\hline & $\mathrm{N}$ & $\%$ & $\mathrm{~N}$ & $\%$ & $\mathrm{~N}$ & $\%$ & $\mathrm{~N}$ & $\%$ \\
\hline $\begin{array}{l}\text { Au moins un dans le même immeuble ou } \\
\text { dans le même quartier }\end{array}$ & 361 & 83,2 & 61 & 14,1 & 12 & 2,8 & 434 & 21,3 \\
\hline $\begin{array}{l}\text { Au moins un dans la région du Pirée/ } \\
\text { Grand Athènes }\end{array}$ & 391 & 37,9 & 524 & 50,8 & 116 & 11,3 & 1031 & 50,7 \\
\hline Aucun dans le Grand Athènes & 4 & 0,7 & 65 & 11,4 & 500 & 87,9 & 569 & 28,0 \\
\hline Total & 756 & 37,1 & 650 & 32,0 & 628 & 30,9 & 2034 & 100,0 \\
\hline
\end{tabular}

b) L'aide dans la vie quotidienne

Nos données ne nous permettent pas un examen approfondi du rôle de la parentèle dans le fonctionnement de la vie familiale quotidienne - les données concernent l'apport des parents depuis le mariage de leurs enfants sans autres précisions sur le contenu de ces aides - (Coenen, J. et al. 1994). Mais la famille grecque, très présente dans les moments importants de la vie comme le mariage et l'installation du jeune couple, l'est aussi dans de nombreuses pratiques de la vie quotidienne (garde des enfants, préparation des repas, aide financière, etc).

Plus d'une famille sur deux a recours aux parents pour « la garde des enfants d'âge préscolaire » (56\%). Trois familles sur dix bénéficient d'une " aide financière », d'un don (32\%). Un tiers également reçoit « une aide domestique » dans la vie quotidienne (35\%: courses, préparation des repas, ménage, etc.). Enfin, $28 \%$ des familles reçoit « d'autres aides importantes ».

Cependant, sur dix familles interrogées, quatre n'ont reçu aucune aide depuis leur mariage. Un tiers environ a reçu 1-2 « aides » et le tiers restant 3-4 « aides », le nombre moyen des « aides reçues » étant au total 1,6 « aides » (1,6 pour les femmes et 1,5 pour les hommes) (tableau 5). Le sexe ne joue donc pas sur l'ampleur de l'aide. Cela montre que l'idée qu'ont les deux époux de cette aide est la même. 


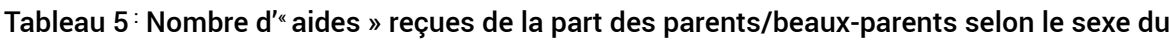
répondant (\% en ligne)

\section{BIBLIOGRAPHIE}

Benoît-Guilbot O., Maratou-Alipranti L., Papliakou V., Sorokos E., Tsanira E., Hadjiyanni A., 1998. Processus de transformation au Pirée : mobilités, famille, emploi. Athènes : Centre National de Recherches Sociales (en grec).

Coenen, J. et al. 1994. Les réseaux de solidarité dans la famille. Lausanne : Réalités Sociales.

Damianakos, S. (dir.) 1987. Processus de changement social en Grèce rurale. Athènes : Centre National de Recherches Sociales (en grec).

Déchaux, J.-H. 1994. « Les échanges de la parenté accentuent-ils les inégalités ? », Sociétés Contemporaines, No 17, p. 75-90.

Degenne, A, Lebeaux, M.-O. 1991. "L'entraide entre les ménages : un facteur d'inégalité sociale ?». Sociétés Contemporaines. No 8, p. 21-42.

Degenne, A, Lebeaux, M.-O. 1993. « Les rôles conjugaux dans leur environnement social ", L'Année Sociologique, No 43, p. 253-268.

Economou, D., Maloutas, T. (dir.) 1988. Problèmes de développement de l'État providence en Grèce. Athènes : Exantas (en grec).

Forsé M., 1991. « Les réseaux de sociabilité : un état des lieux ». L'Année Sociologique, No 41, p. 247-264.

Forsé M., 1993. « La fréquence des relations de sociabilité : typologie et évolution », L'Année Sociologique, No 43, p. 189-212.

Getimis, P. , Gravaris, D. (dir.). 1993. État social et politique sociale. Athènes : Themelio (en grec).

Gilland, P., Levy, M., (dir.), (1990), Famille et solidarité dans une société en mutation, Lausanne : Réalités sociales.

Kayser, B., (1968), « Les migrations interieures en Grèce », in Peristiany, J., (dir.), Contributions to Mediterranean Sociology. Mouton : The Hague, p. 191-200.

Kayser, B, Pechoux, P. Y., Sivignon, M. 1971. Exode rural et attraction urbaine en Grèce. Athènes : Centre National de Recherches Sociales (EKKE).

Kotzamanis V., 1997. « Athènes, 1848-1995. L'émergence démographique d'une métropole ». The Greek Review of Social Research, No 92-93, p. 3-30.

Maloutas, T., 1990. Logement et famille à Athènes. Athènes : Exantas (en grec).

Maloutas, T. 1995. « Ségrégation urbaine et relations familiales dans deux villes grecques: Athènes et Volos ». Sociétés Contemporaines, No 22-23, p. 89-106.

Maratou-Alipranti, L., 1993. "The urbanisation process in Greece : the case of Athens" in Polarisation and Urban Space. Cross-National Research Paper: Loghoborough University, p. 54-64. 
Maratou-Alipranti, L., Papliakou V., Hadjiyanni A., 1995. « Réseaux sociaux en milieu urbain : sociabilité, entraide, emploi ». The Greek Review of Social Research, No 88, p. 172-211.

\section{ANNEXES}

\begin{tabular}{|l||l||l|l|l|l|l|l|l|l|}
\hline & \multicolumn{1}{|l|}{ aucune aide } & $1-2$ aides & $3-4$ aides & moyenne & \multicolumn{2}{l|}{$\begin{array}{l}\text { Total } \\
\text { des aides }\end{array}$} \\
\hline $\mathrm{N}$ & $\%$ & $\mathrm{~N}$ & $\%$ & $\mathrm{~N}$ & $\%$ & & & $\mathrm{~N}$ & $\%$ \\
\hline Hommes & 139 & 38,2 & 127 & 34,9 & 98 & 26,9 & 1,4 & 364 & 100,0 \\
\hline Femmes & 139 & 34,6 & 139 & 34,6 & 124 & 30,8 & 1,6 & 402 & 100,0 \\
\hline \hline Total & 278 & 36,3 & 266 & 34,7 & 222 & 29,0 & 1,5 & 766 & 100,0 \\
\hline
\end{tabular}

\section{NOTES}

1. Benoît-Guilbot O. et al. (1998).

2. Kaminia, Drapetsona, Pérama et Kastella.

\section{RÉSUMÉS}

Cet article présente une partie des matériaux réunis lors des recherches de sociologie urbaine réalisées dans le cadre d'une collaboration franco-hellénique entre 1986 et 1992 (Cnrs, Ekke grec et université de Paris X-Nanterre). L'enquête empirique s'est déroulée dans quatre quartiers de la ville du Pirée, trois quartiers ouvriers et un quartier petit-bourgeois, auprès d'un échantillon représentatif de huit cents individus (dont la moitié de femmes), d'âge compris entre 20 et 59 ans. Menée par questionnaire, cette investigation visait à recueillir des données portant essentiellement sur la famille, la mobilité socio-professionnelle et l'emploi. L'analyse présentée ici est plus particulièrement centrée sur deux points : d'une part, les formes de relations nouées entre générations à l'intérieur de la famille et, d'autre part, les types de sociabilité qui se développent au niveau de la localité. Elle révèle un décalage prononcé opposant les pratiques et les mentalités des Piréotes de souche à celles des « immigrés de l'intérieur » qui, des années après leur installation en ville, entretiennent beaucoup plus de relations avec leur village d'origine qu'avec leur quartier d'adoption.

This article presents material collected through urban sociological research carried out by a Franco-hellenic collaboration between 1986 and 1992 (CNRS, Greek EKKE and université de Paris $\mathrm{X}$-Nanterre). The empirical survey was spread over four district in Piraeus, which are three 
quarters working class and one quarter petty bourgeois, using a representative sample of 800 individuals (50\% women) between the ages of 20 and 59. This investigation, done by questionnaire, aimed to collect data concerning the family, socio-professional mobility and employment. The analysis presented here focuses on two aspects: on the one hand, the types of relations between generations inside the family and, on the other hand, the types of sociabilities which develop in the community. It reveals a distinct gap between the practices and attitudes of original inhabitants of Piraeus and those of immigrants from the "interior" who, after years of settling in the town, maintain greater connections with their village of origin than with their district of adoption.

INDEX

Index géographique : Grèce

Mots-clés : Ville

\section{AUTEURS}

\section{LAURA MARATOU-ALIPRANTI}

Centre National de Recherches Sociales (EKKE), Athènes 